\title{
INTUITIONISTIC FUZZY LINEAR FRACTIONAL PROGRAMMING PROBLEM USING DENOMINATOR OBJECTIVE RESTRICTION METHOD
}

\author{
S. Narayanamoorthy ${ }^{1} \S$, P. Jayaraman ${ }^{2}$, D. Meera ${ }^{3}$ \\ $1,2,3$ Department of Mathematics \\ Bharathiar University \\ Coimbatore, 641 046, INDIA
}

\begin{abstract}
The present paper describes for solving intuitionistic fuzzy linear fractional programming problem where subject to the constraints of right hand side is symmetric trapezoidal intuitionistic fuzzy number. To rank the symmetric trapezoidal intuitionistic fuzzy number by using ranking function. The denominator objective restriction method for finding an optimal solution to the intuitionistic fuzzy linear fractional programming problem. The proposed procedure is illustrated with a numerical example. To find the membership function and nonmembership function of $(\alpha, \beta)$-cut method by using MATLAB.
\end{abstract}

AMS Subject Classification: 90C05, 90C30, 90C70, 90C90

Key Words: linear fractional programming problem, intuitionistic fuzzy set, intuitionistic fuzzy number, ranking function, denominator objective restriction method, intuitionistic fuzzy linear fractional programming problem

\section{Introduction}

In mathematical optimization, linear-fractional programming (LFP) is a generalization of linear programming (LP). Whereas the objective function in a

Received: December 20, 2016

Revised: $\quad$ April 18, 2017

Published: $\quad$ June 7,2017

(c) 2017 Academic Publications, Ltd. url: www.acadpubl.eu

${ }^{\S}$ Correspondence author 
linear program is a linear function, the objective function in a linear-fractional program is a ratio of two linear functions. The linear fractional programming are used as application in many important fields such as production planning, financial and corporate planning, health care and hospital planning. In 1960, Hungarian and Bela Martos ([5], [6]) formulated the linear fractional programming problem. Charnes and Cooper 1962 [3] developed a linear fractional functionals by resolving it into two linear programming problems. Swarup 1965 [14] introduced a simplex technique for the linear fractional programming problem. J. K. Sharma, A. K. Gupta and M. P. Gupta 1980 [15] solved a linear fractional functional programming problem replacing three basic variables by three nonbasic variables at a time by using the simplex technique. The field of Fuzzy set theory was formalized by Professor Lofti Zadeh in 1965 [16] to represent or manipulate data and information possessing non statistical uncertainties. Bellman and Zadeh (1970) [2] provides the concept of decision making in fuzzy environments. Zimmermann 1991 [17] describes the formulation of fuzzy linear programming problem. Pandian and Jayalakshmi 2013 [11] proposed for finding an optimal fuzzy solution to the fully fuzzy linear fractional programming problem by using denominator objective restriction method. Nachammai and Thangaraj 2012 [7] solved a fuzzy linear fractional programming problem where the parameters and variables are triangular fuzzy numbers by using ranking method based on metric distance. Ganesan and Veeramani 2006 [4] provides the fuzzy arithmetic for symmetric trapezoidal fuzzy numbers and proposed a method for solving fuzzy linear programming problems. The intuitionistic fuzzy set theory is an extension of the fuzzy set theory by Atanassov 1986 [1]. Nagoorgani and Ponnalagu 2012 [8] introduced the accuracy function to defuzzify the triangular intuitionistic fuzzy number and to solved the intuitionistic fuzzy linear programming is obtained. Nagoorgani and Ponnalagu 2013 [9] introduced single step algorithm to solved a intuitionistic fuzzy linear programming problem with triangular fuzzy number. Parvathi and Malathi [13] introduced a symmetric trapezoidal intuitionistic fuzzy number (STIFN) and their desirable properties are also included. A new type of intuitionistic fuzzy arithmetic operations for STIFN have been proposed based on $(\alpha, \beta)$-cuts. Parvathi and Malathi proposed the intuitionistic fuzzy linear programming problem using symmetric trapezoidal intuitionistic fuzzy number and a ranking function is presented in this paper to rank STIFN.

In this paper introduces a linear fractional programming problem with intuitionistic fuzzy number. The intuitionistic fuzzy linear fractional programming problem is that in which the objective function is the ratio of numerator and denominator. To find the intuitionistic fuzzy linear fractional programming 
problem by using denominator objective restriction method. The denominator objective restriction method is based on the intuitionistic fuzzy simplex method.

This paper is organized as follows: In Section 2 provides the basic definitions and notations of IFS, IFN, TIFN, STIFN. In Section 3 some formulation of the problem are recalled. In Section 4 describes the algorithm for Denominator Objective Restriction Method. In Section 5, the proposed method is illustrated with a numerical example. In Section 6, finally follows the conclusion.

\section{Preliminaries}

Definition 2.1. (Intuitionistic Fuzzy Set) An Intuitionistic Fuzzy Set (IFS) $\mathrm{A}$ in $\mathrm{X}$ is defined as an object of the form $A=\left\{\left\langle x, \mu_{A}(x), \nu_{A}(x)\right\rangle: x \in X\right\}$ where the functions $\mu_{A}: X \rightarrow[0,1]$ and $\nu_{A}: X \rightarrow[0,1]$ define the degree of membership and the degree of nonmembership of the element $x \in X$ respectively, and for every $x \in X$ in $\mathrm{A}, 0 \leq \mu_{A}(x)+\nu_{A}(x) \leq 1$ holds.

Definition 2.2. (Intuitionistic Fuzzy Number) An Intuitionistic Fuzzy Number (IFN) $\widetilde{A}^{I}$ is:

(i) an intuitionistic fuzzy subset of the real line,

(ii) normal, that is, there is some $x_{0} \in R$ such that $\mu_{\widetilde{A}^{I}}\left(x_{0}\right)=1, \nu_{\widetilde{A}^{I}}\left(x_{0}\right)=$ 0

(iii) convex for the membership function $\mu_{\widetilde{A}^{I}}(x)$, that is

$$
\mu_{\widetilde{A}^{I}}\left(\lambda x_{1}+(1-\lambda) x_{2}\right) \geq \min \left(\mu_{\widetilde{A}^{I}}\left(x_{1}\right), \mu_{\widetilde{A}^{I}}\left(x_{2}\right)\right),
$$

for every $x_{1}, x_{2} \in R, \lambda \in[0,1]$,

(iv) concave for the nonmembership function $\nu_{A}(x)$, that is

$$
\nu_{\widetilde{A}^{I}}\left(\lambda x_{1}+(1-\lambda) x_{2}\right) \leq \max \left(\nu_{\widetilde{A}^{I}}\left(x_{1}\right), \nu_{\widetilde{A}^{I}}\left(x_{2}\right)\right),
$$

for every $x_{1}, x_{2} \in R, \lambda \in[0,1]$.

Definition 2.3. (Trapezoidal Intuitionistic Fuzzy Number) A Trapezoidal Intuitionistic Fuzzy Number (TIFN) $\widetilde{A}^{I}$ is an IFS in R with membership function and nonmembership function as follows:

$$
\mu_{\widetilde{A}^{I}}(x)= \begin{cases}\frac{x-\left(a_{1}-\alpha\right)}{\alpha} & \text { for } x \in\left[a_{1}-\alpha, a_{1}\right] \\ 1 & \text { for } x \in\left[a_{1}, a_{2}\right] \\ \frac{a_{2}+\beta-x}{\beta} & \text { for } x \in\left[a_{2}, a_{2}+\beta\right] \\ 0 & \text { otherwise; }\end{cases}
$$




$$
\nu_{\widetilde{A}^{I}}(x)= \begin{cases}\frac{a_{1}-x}{\alpha^{\prime}} & \text { for } x \in\left[a_{1}-\alpha^{\prime}, a_{1}\right] \\ 0 & \text { for } x \in\left[a_{1}, a_{2}\right] \\ \frac{x-a_{2}}{\beta^{\prime}} & \text { for } x \in\left[a_{2}, a_{2}+\beta^{\prime}\right] \\ 1 & \text { otherwise, }\end{cases}
$$

where $\tilde{A}^{I}=\left[a_{1}, a_{2}, \alpha, \beta ; a_{1}, a_{2}, \alpha^{\prime}, \beta^{\prime}\right]$.

Definition 2.4. (Symmetric Trapezoidal Intuitionistic Fuzzy Number) A TIFN is said to be STIFN if $\alpha=\beta$ (say h) and $\alpha^{\prime}=\beta^{\prime}$ (say $h^{\prime}$ ). Hence the definition of STIFN is as follows:

An IFS $\widetilde{A}^{I}$ in $\mathrm{R}$ is said to be a Symmetric Trapezoidal Intuitionistic Fuzzy Number (STIFN) if there exist real numbers $a_{1}, a_{2}, h, h^{\prime}$ where $a_{1} \leq a_{2}, h \leq h^{\prime}$ and $h, h^{\prime}>0$ such that the membership and nonmembership functions are as follows:

$$
\begin{aligned}
& \mu_{\widetilde{A}^{I}}(x)= \begin{cases}\frac{x-\left(a_{1}-h\right)}{h} & \text { for } x \in\left[a_{1}-h, a_{1}\right] \\
\frac{1}{\frac{a_{2}+h-x}{h}} & \text { for } x \in\left[a_{1}, a_{2}\right] \\
0 & \text { for } x \in\left[a_{2}, a_{2}+h\right]\end{cases} \\
& \nu_{\widetilde{A}^{I}}(x)= \begin{cases}\frac{a_{1}-x}{h^{\prime}} & \text { for } x \in\left[a_{1}-h^{\prime}, a_{1}\right] \\
0 & \text { for } x \in\left[a_{1}, a_{2}\right] \\
\frac{x-a_{2}}{h^{\prime}} & \text { for } x \in\left[a_{2}, a_{2}+h^{\prime}\right] \\
1 & \text { otherwise, }\end{cases}
\end{aligned}
$$

where $\tilde{A}^{I}=\left[a_{1}, a_{2}, h, h ; a_{1}, a_{2}, h^{\prime}, h^{\prime}\right]$.

\section{Formulation of the Problem}

\subsection{Linear Fractional Programming Problem}

The Linear Fractional Programming Problem can be formulated as follows:

Given objective function

$$
Q(x)=\frac{P(x)}{D(x)}=\frac{\sum_{j=1}^{n} p_{j} x_{j}+p_{0}}{\sum_{j=1}^{n} d_{j} x_{j}+d_{0}},
$$


which must be maximized (or minimized) subject to

$$
\left.\begin{array}{rl}
\sum_{j=1}^{n} a_{i j} x_{j} \leq b_{i}, & i=1,2, \ldots, m_{1}, \\
\sum_{j=1}^{n} a_{i j} x_{j} \geq b_{i}, & i=m_{1}+1, m_{1}+2, \ldots, m_{2}, \\
\sum_{j=1}^{n} a_{i j} x_{j}=b_{i}, \quad i=m_{2}+1, m_{2}+2, \ldots, m . & \\
x_{j} \geq 0, \quad j=1,2, \ldots, n_{1},
\end{array}\right\}
$$

where $m_{1} \leq m_{2} \leq m, n_{1} \leq n$. Here and in what follows we suppose that $D(x) \neq 0, \forall x=\left(x_{1}, x_{2}, \ldots, x_{n}\right) \in S$, where $\mathrm{S}$ denotes a feasible set or set of feasible solutions defined by constraints (2)-(3).

\subsection{Fuzzy Linear Fractional Programming Problem}

Fuzzy linear fractional programming problem is defined as:

$$
\begin{gathered}
\text { Maximize }(\tilde{C} \tilde{X}+\alpha) /(\tilde{D} \tilde{X}+\beta)=\tilde{R}(\tilde{X}), \\
\text { subject to the constraints: } \tilde{A} \tilde{X} \leq \tilde{b}, \\
\tilde{X} \geq \tilde{0} .
\end{gathered}
$$

With additional assumption: the denominator is positive for all possible solutions.

\subsection{Intuitionistic Fuzzy Linear Fractional Programming Problem}

The intuitionistic fuzzy set theory is an extension of the fuzzy set theory by Atanassov [1]. The intuitionistic fuzzy linear fractional programming problem (IFLFPP) is a special type of fuzzy linear fractional programming problem (FLFPP). In real life, information available for certain situations is vague and such uncertainty is unavoidable. The possible solution is to consider the parameters involved as intuitionistic fuzzy data.

Consider the Intuitionistic Fuzzy Linear Fractional Programming Problem

$$
\text { Maximize: } \tilde{Z}^{I}=\frac{\left(\tilde{C}^{I} \tilde{X}^{I}+\alpha\right)}{\left(\tilde{D}^{I} \tilde{X}^{I}+\beta\right)},
$$

subject to the constraints: $\tilde{A}^{I} \tilde{X}^{I} \leq \tilde{b}^{I}$,

$$
\tilde{X}^{I} \geq \tilde{0}^{I} \text {. }
$$




\subsection{Ranking Function}

Let $\Re$ be any linear ranking function. Then:

(i) $\tilde{A}^{I} \geq \tilde{B}^{I}$ if and only if $\tilde{A}^{I}-\tilde{B}^{I} \geq 0$ iff $-\tilde{B}^{I} \geq-\tilde{A}^{I}$.

(ii) If $\tilde{A}^{I} \geq \tilde{B}^{I}$ and $\tilde{C}^{I} \geq \tilde{D}^{I}$, then $\tilde{A}^{I}+\tilde{C}^{I} \geq \tilde{B}^{I}+\tilde{D}^{I}$.

Since there are many ranking functions for comparing IF numbers, a linear ranking function is introduced in this paper which is actually a ranking function $\Re$ such that

$$
\Re\left(K \tilde{A}^{I}+\tilde{B}^{I}\right)=K \Re\left(\tilde{A}^{I}\right)+\Re\left(\tilde{B}^{I}\right) .
$$

Below a method of ranking STIFN is suggested which is actually a direct method for ranking classical intuitionistic fuzzy numbers.

$$
\Re\left(\tilde{A}^{I}\right)=a_{1}+a_{2}+\frac{1}{2}\left(h^{\prime}-h\right),
$$

where $\tilde{A}^{I}=\left[a_{1}, a_{2}, h, h ; a_{1}, a_{2}, h^{\prime}, h^{\prime}\right] \in F(R)$.

\section{Algorithm for Denominator Objective Restriction Method}

Step 1: Consider the Intuitionistic Fuzzy Linear Fractional Programming Problem

$$
\text { Maximize: } \tilde{Z}^{I}=\frac{\left(\tilde{C}^{I} \tilde{X}^{I}+\alpha\right)}{\left(\tilde{D}^{I} \tilde{X}^{I}+\beta\right)},
$$

subject to the constraints: $\tilde{A}^{I} \tilde{X}^{I}=\tilde{b}^{I}$,

$$
\tilde{X}^{I} \geq \tilde{0}^{I}
$$

Step 2: Constract two single objective intuitionistic fuzzy linear programming problems namely, the problem $(\mathrm{N})$ as well as the problem (D) from the given problem.

Step 3: Compute to the optimal solution to the problem $(\mathrm{N})$ by means of the intuitionistic fuzzy simplex method. Introduce slack variables to convert the inequalities into equations and let the optimal solution to the problem $(\mathrm{N})$ be $\tilde{X}_{0}^{I}$ and $\operatorname{Max} \tilde{Z}^{I}\left(\tilde{X}_{0}^{I}\right)=\tilde{Z}_{0}^{I}$.

Step 4: Using the optimal table of the problem $(\mathrm{N})$ as an intial intuitionistic fuzzy simplex table to the problem (D), continue to find a sequence of improved 
basic fessible solutions $\left\{\tilde{X}_{n}^{I}\right\}$ to the problem (D) and the value of $\tilde{Z}^{I}$ at each of the imroved basic feasible solution by the intuitionistic fuzzy simplex method.

Step 5: (a) If $\tilde{Z}^{I}\left(\tilde{X}_{k}^{I}\right) \leq \tilde{Z}^{I}\left(\tilde{X}_{k+1}^{I}\right)$ for all $k=0,1,2, \ldots, n-1$ and $\tilde{Z}^{I}\left(\tilde{X}_{n}^{I}\right) \geq \tilde{Z}^{I}\left(\tilde{X}_{n+1}^{I}\right)$ for some $\mathrm{n}$, stop the computation process and then, go to Step 7.

Step 6: (b) If $\tilde{Z}^{I}\left(\tilde{X}_{k}^{I}\right) \leq \tilde{Z}^{I}\left(\tilde{X}_{k+1}^{I}\right)$ for all $k=0,1,2, \ldots, n$ and $\tilde{X}_{n+1}^{I}$ is an optimal solution to the problem (D) for some n, stop the computation process and then, go to Step 8.

Step 7: $\tilde{X}_{n}^{I}$ is an optimal solution to the given problem and $\operatorname{Max} \tilde{Z}^{I}\left(\tilde{X}^{I}\right)=$ $\tilde{Z}^{I}\left(\tilde{X}_{n}^{I}\right)$.

Step 8: $\tilde{X}_{n+1}^{I}$ is an optimal solution to the given problem and $\operatorname{Max} \tilde{Z}^{I}\left(\tilde{X}^{I}\right)$ $=\tilde{Z}^{I}\left(\tilde{X}_{n+1}^{I}\right)$.

\section{Numerical Example}

Consider the following IFLFP problem

$$
\begin{aligned}
\text { Maximize: } & \tilde{Z}^{I}=\frac{6 \tilde{x}_{1}^{I}+3 \tilde{x}_{2}^{I}+6}{5 \tilde{x}_{1}^{I}+2 \tilde{x}_{2}^{I}+5} \\
\text { subject to: } & 4 \tilde{x}_{1}^{I}-2 \tilde{x}_{2}^{I} \leq[8,11,2,2 ; 8,11,4,4], \\
& 3 \tilde{x}_{1}^{I}+5 \tilde{x}_{2}^{I} \leq[10,14,4,4 ; 10,14,6,6], \\
& \tilde{x}_{1}^{I}, \tilde{x}_{2}^{I} \geq 0 .
\end{aligned}
$$

The following two IFLFP problems can be obtained from the given problem:

(N) Maximize $P\left(\tilde{X}^{I}\right)=6 \tilde{x}_{1}^{I}+3 \tilde{x}_{2}^{I}+6$

subject to

$$
\begin{aligned}
& 4 \tilde{x}_{1}^{I}-2 \tilde{x}_{2}^{I} \leq[8,11,2,2 ; 8,11,4,4] \\
& 3 \tilde{x}_{1}^{I}+5 \tilde{x}_{2}^{I} \leq[10,14,4,4 ; 10,14,6,6] \\
& \text { and } \tilde{x}_{1}^{I}, \tilde{x}_{2}^{I} \geq 0
\end{aligned}
$$

(D) Minimize $Q\left(\tilde{X}^{I}\right)=5 \tilde{x}_{1}^{I}+2 \tilde{x}_{2}^{I}+5$

subject to

$$
\begin{aligned}
& 4 \tilde{x}_{1}^{I}-2 \tilde{x}_{2}^{I} \leq[8,11,2,2 ; 8,11,4,4] \\
& 3 \tilde{x}_{1}^{I}+5 \tilde{x}_{2}^{I} \leq[10,14,4,4 ; 10,14,6,6] \\
& \text { and } \tilde{x}_{1}^{I}, \tilde{x}_{2}^{I} \geq 0
\end{aligned}
$$

The standard form of the intuitionistic fuzzy linear programming problem becomes 


$$
\begin{aligned}
\mathrm{N} \Rightarrow \operatorname{Max} P\left(\tilde{X}^{I}\right)= & 6 \tilde{x}_{1}^{I}+3 \tilde{x}_{2}^{I}+0 \tilde{x}_{3}^{I}+0 \tilde{x}_{4}^{I}+6 \\
\text { subject to } & \\
& 4 \tilde{x}_{1}^{I}-2 \tilde{x}_{2}^{I}+\tilde{x}_{3}^{I}=[8,11,2,2 ; 8,11,4,4] \\
& 3 \tilde{x}_{1}^{I}+5 \tilde{x}_{2}^{I}+\tilde{x}_{4}^{I}=[10,14,4,4 ; 10,14,6,6] \\
& \tilde{x}_{1}^{I}, \tilde{x}_{2}^{I}, \tilde{x}_{3}^{I}, \tilde{x}_{4}^{I} \geq 0
\end{aligned}
$$

where $\tilde{x}_{3}^{I}, \tilde{x}_{4}^{I}$ are the slack intuitionistic fuzzy variables.

The optimal solution to the problem $(\mathrm{N})$, by the intuitionistic fuzzy simplex method, is given by the following table:

Table 1

\begin{tabular}{|c|c|c|c|c|c|c|c|c|}
\hline \multicolumn{2}{|c|}{ Basic Variables } & $\tilde{C}_{j}^{I}$ & 6 & 3 & 0 & 0 & & \\
\hline$\tilde{C}_{B}^{I}$ & $\tilde{Y}_{B}^{I}$ & $\tilde{X}_{B}^{I}$ & $\tilde{x}_{1}^{I}$ & $\tilde{x}_{2}^{I}$ & $\tilde{x}_{3}^{I}$ & $\tilde{x}_{4}^{I}$ & $\Re$ & $\theta$ \\
\hline 0 & $\tilde{x}_{3}^{I}$ & {$[8,11,2,2 ; 8,11,4,4]$} & 4 & -2 & 1 & 0 & 20 & 5 \\
\hline 0 & $\tilde{x}_{4}^{I}$ & {$[10,14,4,4 ; 10,14,6,6]$} & 3 & 5 & 0 & 1 & 25 & 8.333 \\
\hline \multicolumn{7}{|c}{$\tilde{P}_{j}^{I}$} \\
$\tilde{P}_{j}^{I}-\tilde{C}_{j}^{I}$ & -6 & -3 & 0 & 0 &
\end{tabular}

Since there are some $\tilde{P}_{j}^{I}-\tilde{C}_{j}^{I}<0$ the current basic feasible solution is not optimal.

Here $\tilde{x}_{1}^{I}$ is entering variable and $\tilde{x}_{3}^{I}$ is leaving variable. Here $\tilde{x}_{2}^{I}$ is entering

Table 2

\begin{tabular}{|c|c|c|c|c|c|c|c|c|}
\hline \multicolumn{2}{|c|}{ Basic Variables } & $\tilde{C}_{j}^{I}$ & 6 & 3 & 0 & 0 & & \\
\hline$\tilde{C}_{B}^{I}$ & $\tilde{Y}_{B}^{I}$ & $\tilde{X}_{B}^{I}$ & $\tilde{x}_{1}^{I}$ & $\tilde{x}_{2}^{I}$ & $\tilde{x}_{3}^{I}$ & $\tilde{x}_{4}^{I}$ & $\Re$ & $\theta$ \\
\hline 6 & $\tilde{x}_{1}^{I}$ & {$\left[\frac{8}{4}, \frac{11}{4}, \frac{2}{4}, \frac{2}{4} ; \frac{8}{4}, \frac{11}{4}, 1,1\right]$} & 1 & $-\frac{2}{4}$ & $\frac{1}{4}$ & 0 & 5 & - \\
\hline 0 & $\tilde{x}_{4}^{I}$ & {$\left[4, \frac{23}{4}, \frac{5}{2}, \frac{5}{2} ; 4, \frac{23}{4}, 3,3\right]$} & 0 & $\frac{13}{2}$ & $-\frac{3}{4}$ & 1 & 10 & 1.5384 \\
\hline \multicolumn{7}{|c}{$\tilde{P}_{j}^{I}$} \\
$\tilde{P}_{j}^{I}-\tilde{C}_{j}^{I}$ & 0 & $-\frac{12}{4}$ & $\frac{6}{4}$ & 0 & \\
\end{tabular}

variable and $\tilde{x}_{4}^{I}$ is leaving variable.

All $\tilde{P}_{j}^{I}-\tilde{C}_{j}^{I} \geq 0$. The current basic feasible solution is optimal.

Therefore, the optimal solution to the problem $(\mathrm{N})$ is

$$
\begin{array}{ll}
\tilde{x}_{1}^{I}=\left[\frac{30}{13}, \frac{83}{26}, \frac{9}{13}, \frac{9}{13} ; \frac{30}{13}, \frac{83}{26}, \frac{16}{13}, \frac{16}{13}\right] \quad \text { and } \quad \Re\left[\tilde{x}_{1}^{I}\right]=5.7692 \\
\tilde{x}_{2}^{I}=\left[\frac{8}{13}, \frac{23}{26}, \frac{5}{13}, \frac{5}{13} ; \frac{8}{13}, \frac{23}{26}, \frac{6}{13}, \frac{6}{13}\right] \quad \text { and } \quad \Re\left[\tilde{x}_{2}^{I}\right]=1.5385
\end{array}
$$


Table 3

\begin{tabular}{|c|c|c|c|c|c|c|c|c|}
\hline \multicolumn{2}{|c|}{ Basic Variables } & $\tilde{C}_{j}^{I}$ & 6 & 3 & 0 & 0 & & \\
\hline$\tilde{C}_{B}^{I}$ & $\tilde{Y}_{B}^{I}$ & $\tilde{X}_{B}^{I}$ & $\tilde{x}_{1}^{I}$ & $\tilde{x}_{2}^{I}$ & $\tilde{x}_{3}^{I}$ & $\tilde{x}_{4}^{I}$ & $\Re$ & $\theta$ \\
\hline 6 & $\tilde{x}_{1}^{I}$ & {$\left[\frac{30}{13}, \frac{83}{26}, \frac{9}{13}, \frac{9}{13} ; \frac{30}{13}, \frac{83}{26}, \frac{16}{13}, \frac{16}{13}\right]$} & 1 & 0 & $\frac{5}{26}$ & $\frac{1}{13}$ & $\frac{75}{13}$ & \\
\hline 3 & $\tilde{x}_{2}^{I}$ & {$\left[\frac{8}{13}, \frac{23}{26}, \frac{5}{13}, \frac{5}{13} ; \frac{8}{13}, \frac{23}{26}, \frac{6}{13}, \frac{6}{13}\right]$} & 0 & 1 & $-\frac{3}{26}$ & $\frac{\frac{1}{2}}{13}$ & $\frac{20}{13}$ & \\
\hline & & $\begin{array}{c}\tilde{P}_{j}^{I} \\
\tilde{P}_{j}^{I}-\tilde{C}_{j}^{I}\end{array}$ & 0 & $\begin{array}{l}3 \\
0\end{array}$ & $\begin{array}{l}\frac{21}{26} \\
\frac{21}{26}\end{array}$ & $\begin{array}{l}\frac{12}{13} \\
\frac{12}{13}\end{array}$ & & \\
\hline
\end{tabular}

$\operatorname{Max} P\left(\tilde{X}^{I}\right)=45.2308$ and the value of Max $\tilde{Z}^{I}=1.2250$.

Now, by Step 4 of the proposed method, the initial intuitionistic fuzzy simplex table to the problem(D) is given below:

The standard form of the intuitionistic fuzzy linear programming problem of $\mathrm{D}$ becomes

$$
\begin{aligned}
\operatorname{Max} & Q\left(\tilde{X}^{I}\right)=-5 \tilde{x}_{1}^{I}-2 \tilde{x}_{2}^{I}+0 \tilde{x}_{3}^{I}+0 \tilde{x}_{4}^{I}-5 \\
\text { subject to } & \\
& 4 \tilde{x}_{1}^{I}-2 \tilde{x}_{2}^{I}+\tilde{x}_{3}^{I}=[8,11,2,2 ; 8,11,4,4] \\
& 3 \tilde{x}_{1}^{I}+5 \tilde{x}_{2}^{I}+\tilde{x}_{4}^{I}=[10,14,4,4 ; 10,14,6,6] \\
& \tilde{x}_{1}^{I}, \tilde{x}_{2}^{I}, \tilde{x}_{3}^{I}, \tilde{x}_{4}^{I} \geq 0
\end{aligned}
$$

where $\tilde{x}_{3}^{I}, \tilde{x}_{4}^{I}$ are the slack intuitionistic fuzzy variables.

Here $\tilde{x}_{3}^{I}$ is entering variable and $\tilde{x}_{1}^{I}$ is leaving variable.

Table 4

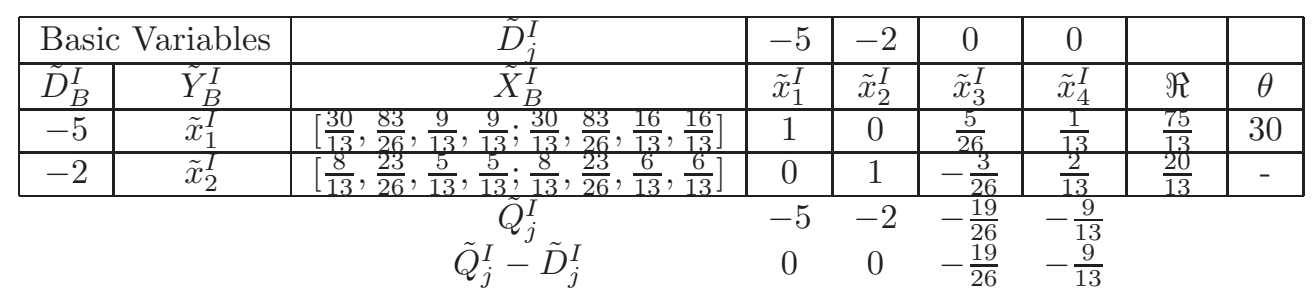

$$
\begin{array}{lll}
\tilde{x}_{1}^{I}=\left[\frac{30}{13}, \frac{83}{26}, \frac{9}{13}, \frac{9}{13} ; \frac{30}{13}, \frac{83}{26}, \frac{16}{13}, \frac{16}{13}\right] & \text { and } \quad \Re\left[\tilde{x}_{1}^{I}\right]=5.7692 \\
\tilde{x}_{2}^{I}=\left[\frac{8}{13}, \frac{23}{26}, \frac{5}{13}, \frac{5}{13} ; \frac{8}{13}, \frac{23}{26}, \frac{6}{13}, \frac{6}{13}\right] & \text { and } \quad \Re\left[\tilde{x}_{2}^{I}\right]=1.5385
\end{array}
$$


$\operatorname{Max} Q\left(\tilde{X}^{I}\right)=-36.923$ and the value of $\tilde{Z}_{0}^{I}=1.2250$. Here $\tilde{x}_{4}^{I}$ is Table 5

\begin{tabular}{|c|c|c|c|c|c|c|c|c|}
\hline \multicolumn{2}{|c|}{ Basic Variables } & $\tilde{D}_{j}^{I}$ & -5 & -2 & 0 & 0 & & \\
\hline$\tilde{D}_{B}^{I}$ & $\bar{Y}_{B}^{I}$ & $\tilde{X}_{B}^{I}$ & $\tilde{x}_{1}^{I}$ & $\tilde{x}_{2}^{I}$ & $\tilde{x}_{3}^{I}$ & $\tilde{x}_{4}^{I}$ & $\Re$ & $\theta$ \\
\hline 0 & $\tilde{x}_{3}^{I}$ & {$\left[12, \frac{83}{5}, \frac{18}{5}, \frac{18}{5} ; 12, \frac{83}{5}, \frac{32}{5}, \frac{32}{5}\right]$} & $\frac{26}{5}$ & 0 & 1 & $\frac{2}{5}$ & 30 & 75 \\
\hline-2 & $\tilde{x}_{2}^{I}$ & {$\left[2, \frac{14}{5}, \frac{4}{5}, \frac{4}{5} ; 2, \frac{14}{5}, \frac{6}{5}, \frac{6}{5}\right]$} & $\frac{3}{5}$ & 1 & 0 & $\frac{1}{5}$ & 5 & 25 \\
\hline & & $\tilde{Q}_{j}^{I}-\tilde{D}_{j}^{I}$ & $\begin{array}{l}-\frac{0}{5} \\
\frac{19}{5}\end{array}$ & $\begin{array}{c}-2 \\
0\end{array}$ & $\begin{array}{l}0 \\
0\end{array}$ & $\begin{array}{l}-\frac{2}{5} \\
-\frac{2}{5}\end{array}$ & & \\
\hline
\end{tabular}

entering variable and $\tilde{x}_{2}^{I}$ is leaving variable.

Table 6

\begin{tabular}{|c|c|c|c|c|c|c|c|c|}
\hline \multicolumn{2}{|c|}{ Basic Variables } & $\tilde{D}_{j}^{I}$ & -5 & -2 & 0 & 0 & & \\
\hline$D_{B}^{I}$ & $Y_{B}^{I}$ & $X_{B}^{I}$ & $\tilde{x}_{1}^{I}$ & $\tilde{x}_{2}^{I}$ & $\tilde{x}_{3}^{I}$ & $\tilde{x}_{4}^{I}$ & $\Re$ & $\theta$ \\
\hline 0 & $\tilde{x}_{3}^{I}$ & {$[8,11,2,2 ; 8,11,4,4]$} & 4 & -2 & 1 & 0 & 20 & \\
\hline 0 & $\tilde{x}_{4}^{I}$ & {$[10,14,4,4 ; 10,14,6,6]$} & 3 & 5 & 0 & 1 & 25 & \\
\hline \multicolumn{7}{|c}{$\tilde{Q}_{j}^{I}$} \\
$\tilde{Q}_{j}^{I}-\tilde{D}_{j}^{I}$ & 5 & 2 & 0 & 0 & & \\
\end{tabular}

All $\tilde{Q}_{j}^{I}-\tilde{D}_{j}^{I} \geq 0$. The current basic feasible solution is optimal. Therefore, the optimal solution to the problem (D) is

$$
\begin{array}{llll}
\tilde{x}_{1}^{I}=0 & \text { and } & \Re\left[\tilde{x}_{1}^{I}\right]=0 \\
\tilde{x}_{2}^{I}=0 & \text { and } & \Re\left[\tilde{x}_{2}^{I}\right]=0
\end{array}
$$

$\operatorname{Max} Q\left(\tilde{X}^{I}\right)=-5$ and the value of $\tilde{Z}_{1}^{I}=1.2$.

Since $\tilde{Z}_{0}^{I}>\tilde{Z}_{1}^{I}$ and by the step $5(\mathrm{a})$ of the proposed method, the optimal solution to the given intuitionistic fuzzy linear fractional programming problem is

$$
\begin{array}{ll}
\tilde{x}_{1}^{I}=\left[\frac{30}{13}, \frac{83}{26}, \frac{9}{13}, \frac{9}{13} ; \frac{30}{13}, \frac{83}{26}, \frac{16}{13}, \frac{16}{13}\right] \quad \text { and } \quad \Re\left[\tilde{x}_{1}^{I}\right]=5.7692 \\
\tilde{x}_{2}^{I}=\left[\frac{8}{13}, \frac{23}{26}, \frac{5}{13}, \frac{5}{13} ; \frac{8}{13}, \frac{23}{26}, \frac{6}{13}, \frac{6}{13}\right] \quad \text { and } \quad \Re\left[\tilde{x}_{2}^{I}\right]=1.5385
\end{array}
$$




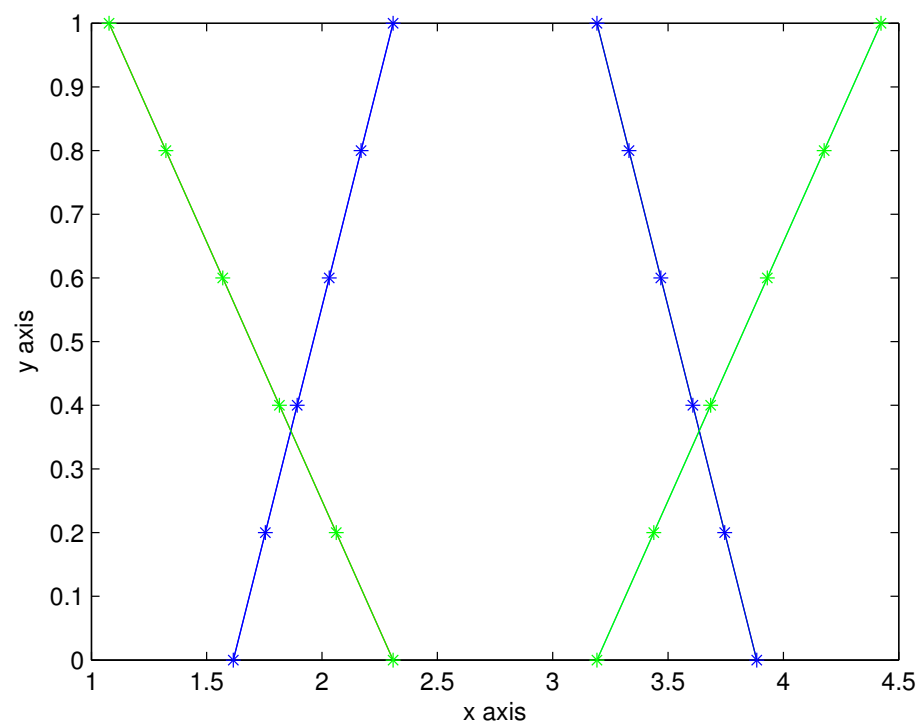

Figure 1: Diagrammatic representation of a STIFN

\begin{tabular}{|c|c|c|c|c|c|}
\hline \multirow{2}{*}{$\alpha$} & \multicolumn{2}{|c|}{$\mu_{\tilde{A}^{I}}(x)$} & \multirow{2}{*}{$\beta$} & \multicolumn{2}{|c|}{$\nu_{\tilde{A}^{I}}(x)$} \\
\cline { 2 - 3 } \cline { 5 - 6 } & $x 1$ & $x 2$ & & $x 3$ & $x 4$ \\
\hline 0 & 1.6154 & 3.8846 & 0 & 2.3077 & 3.1923 \\
\hline 0.2 & 1.7538 & 3.7462 & 0.2 & 2.0615 & 3.4385 \\
\hline 0.4 & 1.8923 & 3.6077 & 0.4 & 1.8154 & 3.6846 \\
\hline 0.6 & 2.0308 & 3.4692 & 0.6 & 1.5692 & 3.9307 \\
\hline 0.8 & 2.1692 & 3.3308 & 0.8 & 1.3231 & 4.1769 \\
\hline 1 & 2.3077 & 3.1923 & 1 & 1.0769 & 4.4231 \\
\hline
\end{tabular}

$$
\operatorname{Max} \quad \tilde{Z}^{I}=1.2250
$$

By using MATLAB the graph will be shown. The membership and nonmembership value of $\tilde{x}_{1}^{I}$ by using $(\alpha, \beta)$-cut method

The membership and nonmembership value of $\tilde{x}_{2}^{I}$ by using $(\alpha, \beta)$-cut method.

\section{Conclusion}

In this paper to define a procedure to solve intuitionistic fuzzy linear fractional programming problem using denominator objective restriction method. The denominator objective restriction method is based on the intuitionistic fuzzy 


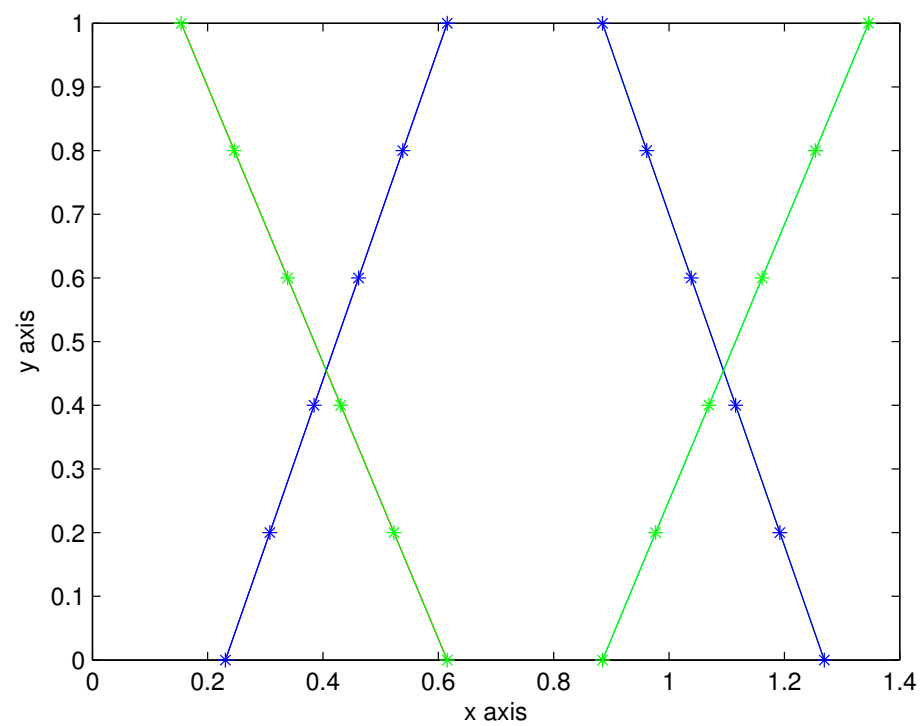

Figure 2: Diagrammatic representation of a STIFN

\begin{tabular}{|c|c|c|c|c|c|}
\hline \multirow{2}{*}{$\alpha$} & \multicolumn{2}{|c|}{$\mu_{\tilde{A}^{I}}(x)$} & \multirow{2}{*}{$\beta$} & \multicolumn{2}{|c|}{$\nu_{\tilde{A}^{I}}(x)$} \\
\cline { 2 - 3 } \cline { 5 - 6 } & $x 1$ & $x 2$ & & $x 3$ & $x 4$ \\
\hline 0 & 0.2308 & 1.2692 & 0 & 0.6154 & 0.8846 \\
\hline 0.2 & 0.3077 & 1.1923 & 0.2 & 0.5231 & 0.9769 \\
\hline 0.4 & 0.3846 & 1.1154 & 0.4 & 0.4308 & 1.0692 \\
\hline 0.6 & 0.4615 & 1.0385 & 0.6 & 0.3385 & 1.1615 \\
\hline 0.8 & 0.5385 & 0.9615 & 0.8 & 0.2462 & 1.2538 \\
\hline 1 & 0.6154 & 0.8846 & 1 & 0.1538 & 1.3462 \\
\hline
\end{tabular}

simplex method. The intuitionistic fuzzy linear fractional programming problem can be reduced to an intuitionistic fuzzy linear programming problem in which the constraints of right hand side is symmetric trapezoidal intuitionistic fuzzy number. This approach is very useful because most theoretical results developed in intuitionistic fuzzy linear programming could be relatively easily expanded to include intuitionistic fuzzy linear fractional programming problems. It is easy to understand, compute and also, to more experiment and industrial applications concerning decision making in an uncertain environment. The methods can serve decision makers by providing an appropriate best solution to a variety of linear fractional programming models having crisp or fuzzy parameters. 


\section{References}

[1] K.T. Atanassov, Intuitionistic fuzzy sets, Fuzzy Sets and Systems, 20 (1986), 87-96.

[2] R.E. Bellman, L.A. Zadeh, Decision making in a fuzzy environment, Management Science, 17 (1970), 141-164.

[3] Charnes A. and Cooper W.W., Programming with linear fractional functional, Nav. Res. Log. Quart., vol. 9, 181-86, (1962).

[4] Ganesan K. and Veeramani P., Fuzzy linear programs with trapezoidal fuzzy numbers, Ann. Oper. Res., vol. 143, 305-315, (2006).

[5] Martos B., Hyperbolic Programming, Publ. Math. Inst., Hungarian Academy of Sciences, vol. 5, Ser. B, 386-406, (1960).

[6] Martos B., Hyperbolic Programming, Naval Research Logistic Quarterly, vol. 11, 135-155, (1964).

[7] Nachammai A. L. and Thangaraj P., Solving fuzzy linear fractional programming problem using metric distance ranking, Applied Mathematical Sciences, vol. 6, 1275-1285, (2012).

[8] Nagoorgani A., Ponnalagu K., A new approach on solving intuitionisitc fuzzy linear programming problem, Applied Mathematical Sciences, vol. 6, 3467-3474, (2012).

[9] Nagoorgani A., Ponnalagu K., An approach to solve intuitionistic fuzzy linear programming problem using single step algorithm, International Journal of Pure and Applied Mathematics, vol. 86, 819-832, (2013).

[10] Narayanamoorthy S. \& Maheswari S.., The Intelligence of Octagonal Fuzzy Number to Determine the Fuzzy Critical Path: A New Ranking Method, Scientific Programming, Vol. 2016, 1-8 pages, (2016).

[11] Pandian P. \& Jayalakshmi M., On Solving Linear Fractional Programming Problems, Published by Canadian Center of Science and Education, Vol. 7, 90-100, (2013).

[12] Parvathi R. \& Malathi C., Intuitionistic Fuzzy Simplex Method, International Journal of Computer Applications, Vol. 48, 39-48, (2012).

[13] Parvathi R. \& Malathi C., Arithmetic Operations on Symmetric Trapezoidal Intuitionistic Fuzzy Numbers, International Journal of Soft Computing and Engineering(IJSCE), Vol. 2, 268-273, (2012).

[14] Swarup K., Linear fractional functional programming, Operation Research, vol. 13, 10291036, (1965).

[15] Sharma J. K., Gupta A. K. and Gupta M. P. Extension of simplex technique for solving fractional programming problem, Indian J. pure appl. Math., vol. 11, 961-968, (1980).

[16] Zadeh L. A., Fuzzy Sets, Information and control, vol. 8, 338-353, (1965).

[17] Zimmermann H. J., Fuzzy Set Theory and its Applications, Kluwer Academic Publishers, (1991). 
